\title{
Study on Dynamic Contact Angles and Capillary Forces Between Two Spherical Particles
}

\author{
Yang Song \\ College of Hydraulic and Architectural Engineering, \\ Yunnan Agricultural University, \\ Kunming, China, 650201 \\ yscliff007@126.com
}

\author{
Cheng Yongjun \\ Zhejiang Dongyang Third Construction Engeering \\ Co.,Ltd, \\ Dongyang, China,322100 \\ movingcock@qq.com
}

\begin{abstract}
Contact angle hysteresis has an important impact on liquid bridges between sphere particles. The paper studies the dynamic contact angles effect on capillary and viscous forces of liquid bridges, calculation results indicate that its effects on capillary force are significant; however, it has little effect on viscous force. Through the mathematical method the capillary force can be expressed as the function of contact angle and filling angle. By fixing the contact angle, changes in the capillary force during the wetting and drying processes can be assessed by analyzing the derived function of such function with regard to the filling angle. By fixing the filling angle, changes in the capillary force in the pinning process can be analyzed using the derived function and the contact angle.
\end{abstract}

Keywords-capillary force; viscous force; contact angle hysteresis

\section{INTRODUCTION}

Particulate matter, such as sand, soil, dust, drugs, chemicals, mining, etc., exists widely in nature and is inseparable from human life. Wet particulate matter is one of the most popular research directions because capillary action complicates the wet particle issue, and the effects of capillary force on wet particles cannot be ignored. Conditions for the existence of a liquid bridge between particles are as follows: when the distance between particles reaches a certain value, the liquid bridge is damaged, and the capillary attraction between particles disappears[1,2]. Pitois[3] considered the effects of contact angles and liquid viscosity, and proposed an energy expression while the liquid bridge is being damaged. Testing has been carried out to verify his proposition. MeqiasAlquacil[9]considered the effects of various factors and conducted analysis on the existing scope of liquid bridges while spaces between particles are increasing. Furthermore, Meqias-Alquacil[4] also discussed the existing limit conditions of liquid bridges while particles are in contact with one another. $\mathrm{Mu}[5]$ and Cai[6]presented a comprehensive summary of studies involving the status of liquid bridges between particles. Luan[7]simplified soil particles using the spherical particle model; tension suction have been proposed through the mechanical analysis of liquid bridges between particles. The model has theoretically derived the soil-water characteristic curve and has established the corresponding strength formula.
This paper considers the contact angle hysteresis effect by assuming that the particles approach or separate in a certain speed under the spherical particle model. The simultaneous equations solving method is adopted to provide a continuous geometric and mechanical variation of liquid bridges. when particles one another or when they separate, changes in contact angle and meniscus radius is continuous and regular. Liquid viscous force cannot be ignored under certain approaching and separating speeds. The contact angle hysteresis effect does not only have a significant impact on capillary force under increasing or decreasing liquid bridge volume, but also significantly affects the mechanical characteristics of liquid bridges during the approaching or separating process.

\section{PARTICLE MODEL ANALYSIS}

\section{A. Geometry analysis}

Two sphere particles are connected by a liquid bridge (Fig $1)$

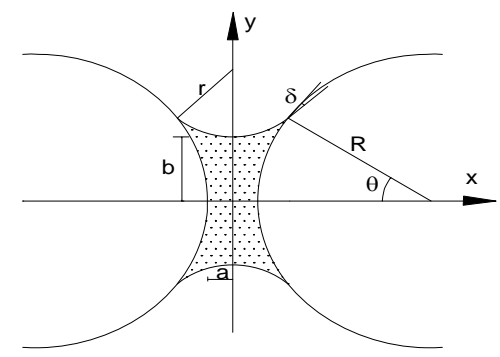

Fig.1 Two particles with a liquid bridge

Let the distance between the sphere particles such that the radius $R$ is equal to $2 a$. $\delta$ is contact angle, $\theta$ is filling angle. The spheres are connected by a liquid bridge (i.e., the meniscus of such liquid bridge complies with thermodynamic assumptions). This liquid bridge is enclosed by two spherical crowns and a circular rotation surface. In the xy axes plane, the liquid surface is a section of the arc with the radius of $r, b$ is the height of the bottom of the liquid surface, and $2(R+a)$ is the distance between the centers of the spheres. The equations that should be satisfied at any point on the meniscus are as follows[7]: 


$$
\begin{gathered}
x^{2}+(y-b-r)^{2}=r^{2} \\
y^{\prime}=-\frac{x}{y-b-r}=\tan \left(\frac{\pi}{2}-\delta-\arcsin \left(\frac{y}{R}\right)\right) \\
(x-R-a)^{2}+y^{2}=R^{2}
\end{gathered}
$$

The volume of the liquid bridge is obtained as follows:

$$
V=2 \pi \int_{0}^{R-R \cos \theta+a}\left(r+b-\sqrt{\left(r^{2}-x^{2}\right)}\right)^{2} d x-2 \pi \int_{a}^{a+R-R \cos \theta}\left(2 R^{2}-x+a\right) d x
$$

\section{B. Mechanical and mathematical analysis}

With the absence of external force, the liquid bridge connects the two sphere particles through Laplace force and tension. Based on the Young-Laplace equation, the force between two sphere particles on the liquid-solid interface is given by

$$
F_{\text {laplace }}=\pi y^{2} \Delta p=\pi y^{2} \sigma\left(\frac{1}{r}-\frac{1}{b}\right)
$$

The water-vapor interface produces tension function on the surface of two particles, and the composite force of the surface tension on the $\mathrm{X}$ direction is given by[7]

$$
F_{t}=2 \pi y \sigma \sin \left(\delta+\arcsin \left(\frac{y}{R}\right)\right)
$$

where $\sigma$ is the surface tension coefficient. The composite force of Laplace force and tension is called capillary force:

$$
F_{\text {cap }}=F_{\text {laplace }}+F_{t}
$$

The functioning scope of $F_{\text {laplace }}$ is the projection of the spherical crown on the y-axis enclosed by the liquid bridge. The distribution force uniformly acting on the spherical crown in the liquid bridge is called matric suction. When $a=0, r$ and $b$ can be expressed as the functions of contact angles $\theta$ and $\delta$ :

$$
\begin{aligned}
& r=R \frac{1-\cos \theta}{\cos (\theta+\delta)} \\
& b=R \tan \theta-r\left(1-\frac{\sin \delta}{\cos \theta}\right)
\end{aligned}
$$

Considering the general situation, Equations (8) and (9) can be expressed as follows:

$$
\begin{aligned}
& r=\frac{R+a-R \cos \theta}{\cos (\theta+\delta)} \\
& b=(R+a) \tan \theta-r\left(1-\frac{\sin \delta}{\cos \theta}\right)
\end{aligned}
$$

According to (10) (11), (5)can be written as

$$
F_{\text {laplace }}=\pi y^{2} \sigma\left[\frac{\cos (\theta+\delta)}{R+a-R \cos \theta}-\frac{\cos \theta \cos (\theta+\delta)}{(R+a)(\sin \theta \cos (\theta+\delta)-(1-\cos \theta)(\cos \theta-\sin \delta))}\right]
$$

Considering the absence of contact angle hysteresis effect, $\delta$ is a constant. Derivation of $\theta$ is carried out in Equation (6):

$$
\frac{d F_{t}}{d \theta}=2 \pi y \sigma \cos (\delta+\theta)
$$

In Equation (13), $\mathrm{y}$ is larger than 0. The range of positive and negative values can be obtained by investigating $\cos (\delta+\theta)$, which can be used to analyze changes in liquid bridge tension along the contact angle, as well as the distance between particles.

Similarly, $\delta$ is fixed(i.e.,without the contact angle hysteresis effect), and derivation of $\theta$ can be carried out in Equation (13). Let its derived function be $F_{1}(\theta)^{\prime}$. R and a are larger than 0 . The changes in Laplace force along $\theta$ can be judged by calculating the positive and negative situation of $F_{1}(\theta)^{\prime}$. If $F_{1}(\theta)^{\prime}$ is 0 , the extreme value of the Laplace force during the changing of a certain section can be calculated. The same method can be used to judge changes in capillary force. The expression of capillary force for the derivative of $\theta$ is as follows:

$$
\frac{d F_{\text {cap }}}{d \theta}=\frac{d F_{t}}{d \theta}+\frac{d F_{\text {laplace }}}{d \theta}
$$

When the contact angle hysteresis effect is considered, $\delta_{\mathrm{r}}<\delta<\delta_{\mathrm{a}}$, and $\theta$ is constant (i.e., $\mathrm{y}$ is kept unchanged, $\delta_{\mathrm{r}}$ is the receding contact angle, $\delta_{\mathrm{a}}$ is the advancing cnotact angle). Derivation of $\theta$ is carried out in Equation (6):

$$
\frac{d F_{t}}{d \delta}=2 \pi y \sigma \cos (\delta+\theta)
$$

When $0<\theta+\delta<\pi / 2$, while the contact angle hysteresis effect occurs, the maximum value is taken at $\delta=\delta_{\mathrm{a}}$. When $\pi / 2<\theta+\delta<\pi$, the maximum value is taken at $\delta=\delta$ r. If $\theta+\delta=\pi / 2$, maximum value is taken at such point. Using the same reasoning, changes in Flaplace and $\mathrm{F}_{\text {cap }}$ along $\delta$ can be judged while the contact angle hysteresis effect occurs. The extreme values of $F_{\text {laplace }}$ and $\mathrm{F}_{\text {cap }}$ are obtained when $\delta$ is changing in a certain section.

\section{VISCOUS FORCE}

While the two sphere particles are in a state of relative movement under certain speed because of liquid viscosity, a viscous force with a direction opposite to the movement speed direction is produced. The size of this viscous force can be determined according to the Reynolds equation:

$$
\frac{d}{d y}\left[y H^{3}(y) \frac{d P(y)}{d y}\right]=12 \eta y \frac{d D}{d t}
$$

Where $D=2 a$, the following can be obtained after integrating both sides of Equation (16):

$$
p(y)=\int \frac{6 \eta y}{H^{3}(y)} \frac{d h}{d t} d y+\int \frac{A}{y H^{3}(y)} d y+B
$$

where $\mathrm{A}$ and $\mathrm{B}$ are the integration constants, $\mathrm{H}(y)=2 a+\mathrm{y}^{2} / \mathrm{R}$. These constants can be determined through the pressure gradient $\partial \mathrm{p} / \partial \mathrm{y}=0$ (when $\mathrm{y}=0$ ) and the boundary condition. If 
$\mathrm{p}(\mathrm{y}) \mid \mathrm{y}=\mathrm{y} 0$ (i.e. the pressure at the boundary is 0$)$, then $\mathrm{p}(\mathrm{y})$ can be obtained from Equation (17):

$$
p(y)=-\frac{3}{2} \pi \eta R^{2}\left[1-\frac{D}{H\left(y_{0}\right)}\right]^{2} \frac{1}{D} \frac{d D}{d t}
$$

If $y_{0}$ is infinite, (18) can be simplified as

$$
p(y)=-\frac{3}{2} \pi \eta R^{2} \frac{1}{D} \frac{d D}{d t}
$$

Equation (19) shows that this equation is not related to the contact angle (i.e., it cannot reflect the impact of contact angle changes on the liquid bridge viscous force). However, in Equation (19), the Reynolds equation is obtained by simplifying the Navier-Stoke equation. The Navier-Stoke equation supposes that the curvature of the gas-liquid interface of the liquid bridge is 0 (i.e., without considering static capillary force), so we can get $\mathrm{p}(\mathrm{y}) \mid \mathrm{y}=\mathrm{y}_{0}=0$. In order to investigate the impacts of static capillary action and the contact angle hysteresis effect on dynamic capillary force [considering that there is very small bending on the gas-liquid interface, and that the fluid pressure is also determined by Equation (19)], the boundary conditions are then changed as follows:

$$
\begin{aligned}
& \left.p(y)\right|_{y=y_{0}}=p_{0} \\
& p(y)=-\frac{3}{2} \pi \eta R^{2}\left[1-\frac{D}{H\left(y_{0}\right)}\right]^{2} \frac{1}{D} \frac{d D}{d t}+\pi p_{0} y_{0}^{2}
\end{aligned}
$$

The $\pi p_{0} \mathrm{y}_{0}^{2}$ in the foregoing equation is the Laplace force; however, the tension does not belong to the pressure inside the liquid; it must be calculated separately. The contact angle hysteresis effect can be reflected approximately by changes in $\mathrm{y}_{0}$. When there is no contact angle hysteresis during the movement process of two particles, $\mathrm{y}_{0}$ changes during the movement process of particles; otherwise, $y_{0}$ does not change.

The contact angle hysteresis effect has very little impact on the viscous force of liquid bridges(Figure2 a\#). When the capillary force is higher than the viscous force, the viscous force can be ignored; otherwise, the capillary force can be ignored. When the speed is in a certain range, the capillary force and viscous force are in the same order; both forces should be considered(Figure $2 \mathrm{~b} \#$ ). Hence, the contact angle hysteresis effect significantly affects viscous force.

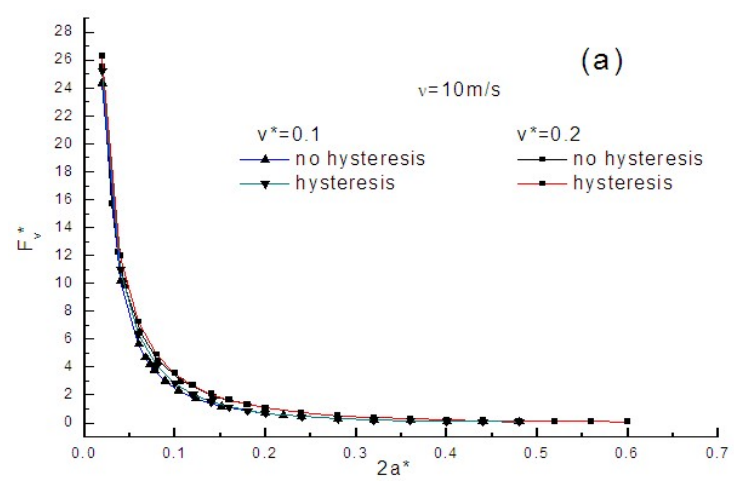

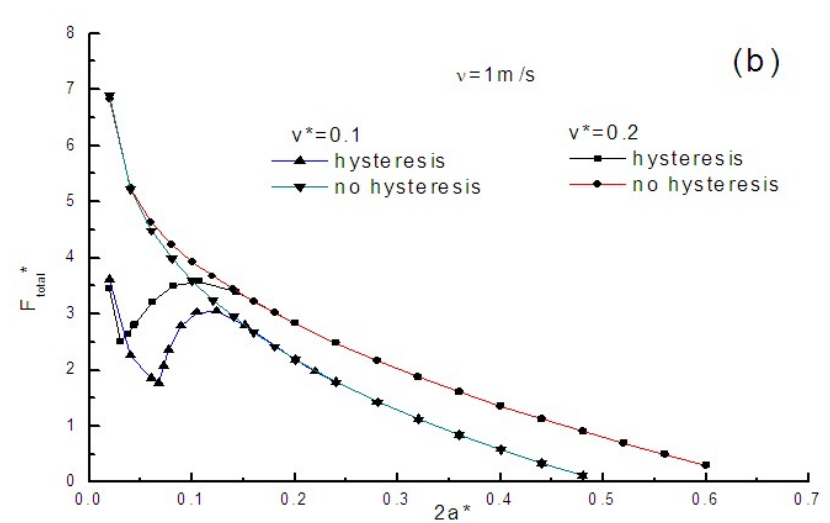

Fig.2 Relative contribution of dimensionless force, $\left(F_{v}, F_{\text {total }}\right)$, as a function of dimensionless separation distance $\left(2 \mathrm{a}^{*}\right)$, (a): viscous force is higher than capillary force, (b): capillary force and viscous force are in the same order ( $\mathrm{F}_{\text {total }}$ dimensionless viscous force and dimensionless capillary force)

\section{CONCLUSIONS}

When the liquid bridge volume between sphere particles is kept unchanged and the two sphere particles are in the state of relative movement, the contact angle hysteresis effect significantly influences capillary force. Capillary force may have its peak value in the section where the contact angle occurs. The changing rule may be obtained by analyzing the derived function of the capillary force for $\delta$ and $\theta$. When the distance between sphere particles is secured and the volume of the liquid is increased (or decreased) or the liquid bridge volume between particles is unchanged and the change in capillary force caused by particle spacing is decreased (or increased). The essence is the change in $\delta$ and $\theta$. The contact angle hysteresis effect is a process which means the continuous increase or decrease of $\delta$, based on fixed $\theta$. When the relative moving speed of the particles is larger than a certain value, the liquid bridge viscous force may be dominant. However, at this time, the contact angle hysteresis effect may produce a negligible impact on the dynamic liquid.

\section{REFERENCES}

[1] Denkov, N. D., Ivanov, I. B. and Kralchevsky P.A. A Possible Mechanism of Stabilization of Emulsions by Solid Particles[J]. J Colloid Interface Sci, 1992,150(2): 589-593.

[2] Lian, G., Thornton, C., and Adams, M. J. A theoretical study of the liquid bridge forces between two rigid spherical bodies[J]. J Colloid interface Sci,1993,161(1):138-147.

[3] Pitois, O., Moucheront, P. and Chateau, X. Rupture energy of pendular liquid bridge[J]. European Physical Journal B, 2001,23(1):79-86.

[4] Meqias-Alquacil, D., Gauckler, L. J. Capillary forces between two solid spheres linked by a concave liquid bridge : Regions of existence and forces mapping[J]. AIChE Journal, 2009,55(5):1103-1109

[5] Mu, F. S., Su, X. B. Analysis of liquid bridge between spherical particles[J]. China Particuology, 2007,5(6):420-424.

[6] Cai, S. B. Bhushan, B. Meniscus and viscous forces during separation of hydrophilic and hydrophobic surface with liquid-mediated contacts[J]. Materials Science and Engineering R, 2008,61: 78-106.

[7] Luan, M.T., Li, S.Q. and Yang, Q. Matric suction, tension suction and their equivalent in four-grain packed unsaturated soils with isolated pore water[J]. Geotechnical Special Publication, 2006,(147):2162-2173. 